\title{
ON THE RECOMBINATION IN THE QUASI-NEUTRAL BASE OF POLYSILICON EMITTER TRANSISTORS WITH INTERFACIAL OXIDES
}

\author{
(Received 26 February 1991; in revised form 23 April 1991)
}

\section{INTRODUCTION}

The current gain of polysilicon emitter transistors can be dramatically increased by introducing an interfacial oxide between the polysilicon and monosilicon parts of the emitter. Although the physical mechanism is not fully understood, this oxide suppresses the injection from the base into the emitter for both npn and pnp devices[1,2].

Ashburn et al. reported that the base current in npn polysilicon emitter bipolar devices with a deliberately grown interfacial oxide is suppressed to such an extent that electron recombination in the quasi-neutral base becomes an important base current component[3]. We have detected base recombination indeed. The strong dependence of the base current on the collector-base voltage reported, however, may be due to impact ionization in the collector-base depletion region.

\section{DEVICE FABRICATION}

The $n p n$ and $p n p$ transistors used in this study were fabricated on separate wafers. The collector was formed by means of high-energy ion implantation. The implantation species for the npn transistors was $P$ with an energy of $1.5 \mathrm{MeV}$ to a dose of $1 \times 10^{13}$ or $2 \times 10^{13} \mathrm{~cm}^{-2}$. For the pnp device, $B$ was used with an energy of $1 \mathrm{MeV}$ to a dose of $3 \times 10^{13} \mathrm{~cm}^{-2}$. The interfacial oxide was made by immersing the wafers in hot $60 \%$ nitric acid for $10 \mathrm{~min}$. Subsequently, a $300 \mathrm{~nm}$ thick undoped polysilicon layer was deposited. The polysilicon was implanted after deposition with arsenic $\left(100 \mathrm{keV}, 4 \times 10^{15} \mathrm{~cm}^{-2}\right)$ or $B\left(40 \mathrm{keV}, 2.5 \times 10^{15} \mathrm{~cm}^{-2}\right)$.

\section{RESULTS}

In Fig. 1, the Gummel plot of the npn device with the higher collector dose is shown for three different collector-base voltages, $0,3.75$ and $4.25 \mathrm{~V}$. The emitter area of this device is $4 \times(25 \times 25) \mu \mathrm{m}^{2}$. The common-emitter current gain is about 2000 at moderate $V_{b e}(0.6-0.7 \mathrm{~V})$. Corresponding devices without a deliberately grown interfacial oxide had typical current gains of about 70 . Thus the interfacial oxide strongly reduces the base current. The collector current is ideal over 7 decades. It varies only a little bit with increasing collector-base voltage $V_{\mathrm{cb}}$. Therefore, it can be concluded that minor extension of the collector-base depletion region into the base occurs for higher $V_{\mathrm{cb}}$. The base current, however, is a strong function of $V_{c b}$. The base current at a $V_{c b}$ of $3.75 \mathrm{~V}$ is considerably lower than that at a zero-biased collector-base. At $V_{c b}=4.25 \mathrm{~V}$, the base current even temporarily changes its sign. This is attributed to impact ionization in the collector-base space charge region[4-6]. At $V_{c b}=4.25 \mathrm{~V}$, the carrier generation in the collector-base depletion region caused by impact ionization can be so heavy that the direction of the base current is opposite. On the other hand, its contribution to the collector current is small.
The field-dependent electron ionization rate may be written as[6,7]:

$$
\alpha_{n}(x)=\alpha_{n}^{0} \exp \left[-\frac{E_{\mathrm{c}}}{E(x)}\right],
$$

where $E(x)$ is the local electrical field. $\alpha_{n}^{0}$ is the avalanche coefficient and $E_{\mathrm{c}}$ the critical electrical field. In simulations presented later on, the following values for $\alpha_{n}^{0}$ and $E_{\mathrm{c}}$ are used: $\alpha_{n}^{0}=7.0 \times 10^{5} \mathrm{~cm}^{-1}$ and $E_{\mathrm{c}}=1.2 \times 10^{6} \mathrm{~V} \mathrm{~cm}^{-1}$.

The extra collector current generated through impact ionization is:

$$
I_{\mathrm{s}}=\int_{a}^{b} I_{\mathrm{c}} \alpha_{n}(x) \mathrm{d} x,
$$

where $a$ and $b$ are the boundaries of the collector-base depletion region. In the area of interest, $I_{c}$ is much larger than $I_{\mathrm{g}}$. Therefore, $I_{\mathrm{c}}$ is assumed to be constant and $I_{\mathrm{g}}$ can be written approximately as:

$$
I_{\mathrm{g}}=\alpha_{n}^{0} I_{\mathrm{c}} \int_{a}^{b} \exp \left[-\frac{E_{\mathrm{c}}}{E(x)}\right] \mathrm{d} x .
$$

From equation (3) an exponential dependence of $I_{g}$ on the collector-base voltage $V_{\mathrm{cb}}$ may be predicted. The impact of $I_{\mathrm{g}}$ on the collector current is of minor importance, but it can affect the base current. The importance of $I_{\text {s }}$ with respect to the base current can be expressed by the following equation:

$$
\Delta_{\mathrm{b} . \mathrm{imp}}=\frac{I_{\mathrm{g}}}{I_{\mathrm{b} 0}}
$$

where $I_{b 0}$ is the base current at the zero-biased collector-base junction. When the collector-base voltage is increased, the depletion layer will further extend into the base and the quasi-neutral base width will decrease. Therefore the recombination of electrons in the quasi-neutral base becomes less with increasing $V_{c b}$. The deviation of the base current, due to a reduced base recombination, is described by:

$$
\Delta_{\mathrm{b} . \mathrm{rec}}=\frac{I_{\mathrm{bo}}-I_{\mathrm{b}}\left(V_{\mathrm{cb}}\right)}{I_{\mathrm{b} 0}}
$$

The total deviation of the base current at a certain $V_{c b}$ follows from the summation of equations (4) and (5). It can be written as:

$$
\Delta_{\mathrm{b}, \mathrm{tot}}=\frac{I_{\mathrm{bb}}-I_{\mathrm{b}}\left(V_{\mathrm{cb}}\right)+I_{\mathrm{g}}}{I_{\mathrm{bO}}} .
$$

Simulations were carried out to investigate the components $\Delta_{\mathrm{b} \text {.imp }}, \Delta_{\mathrm{b} \text {.roc }}$ and $\Delta_{\mathrm{b} \text {. } \mathrm{cot}}$. Since our device simulator does not account for any interfacial oxide, the polysilicon interface was modelled by means of an effective recombination velocity $S_{\mathrm{pi}}$ of $1 \times 10^{3} \mathrm{~cm}^{-1}$. This value corresponds very well with typical values reported elsewhere[8]. The electron recombination in the quasi-neutral base depends on the electron lifetime. In the doping region of interest, the 


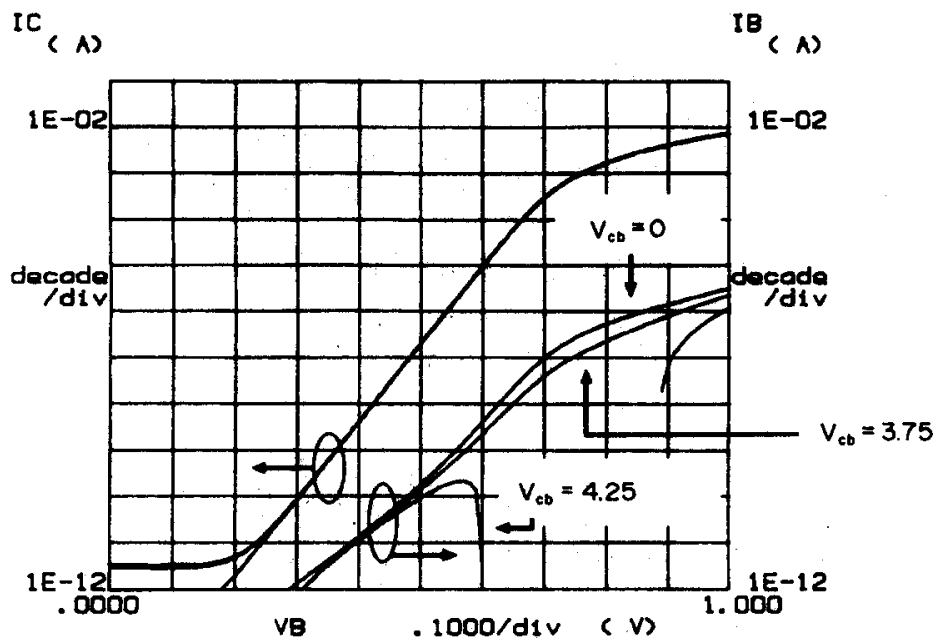

Fig. 1. A Gummel plot of the npn device with the higher collector dose. The collector current hardly varies with increasing $V_{c b}$. On the other hand, the base current is a strong function of $V_{c b}$ due to impact ionization in the collector-base depletion region.

electron lifetime is mainly determined by SRH-recombination and can be written as:

$$
\frac{1}{\tau_{n}}=\frac{1}{\tau_{0}}+\frac{1}{\tau_{\mathrm{SRH}}}=\frac{1}{\tau_{0}}+C_{\mathrm{SRH}} N_{\mathrm{A}}
$$

In our simulations $\tau_{0}$ and $C_{\mathrm{SRH}}$ are $1 \mu \mathrm{s}$ and $1 \times 10^{-11} \mathrm{~cm}^{3}$ $\mathrm{s}^{-1}$, respectively.

For the npn transistor with the higher collector concentration, the simulation results of $\Delta_{b \text {,imp }}, \Delta_{b, \text { rec }}$ and $\Delta_{b \text {. cot }}$ are plotted in Fig. 2. Agreement between the simulated and measured values is good. At low $V_{\text {, }}$, the base current deviation can be attributed to a reduced electron recombination in the quasi-neutral base. The simulations point out that $20 \%$ of the base current originates from electron recombination in the quasi-neutral base. At $V_{c b}$ higher than $2 \mathrm{~V}$, the impact ionization gives rise to a pronounced increase of the base current deviation. It can be observed from the figure that the impact ionization exhibits an exponential behaviour indeed.

In Fig. 3, the relative base current deviation is illustrated including the other $n p n$ and $p n p$ devices. The decrease of the base current, caused by impact ionization in the collector-base depletion region, is already significant at low $V_{c b}$ for the npn devices. Lowering the collector concentration at the collector-base junction, by means of a reduction of the

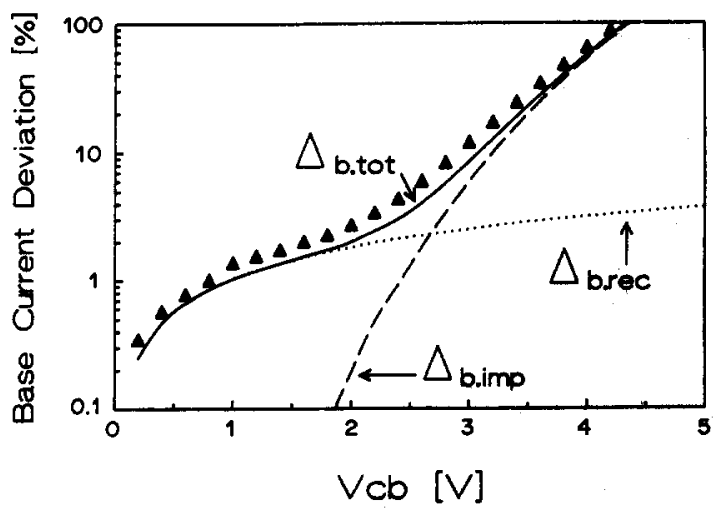

Fig. 2. The simulated and measured base current deviation as a function of the collector-base voltage for the npn device with the high collector dose. The triangles represent the measured data
$P$ implantation dose, results in lower ionization rates at a given $V_{c b}$. For $V_{c b}$ voltages less than $2 \mathrm{~V}$, the base current of the $n p n$ devices varies only little with an increasing $V_{\text {eth }}$. As stated earlier, this is due to electron recombination in the quasi-neutral base. Compared to the decrease of the base current, as reported by Ashburn et al.[3], the reduction due to base recombination is small in our devices. However, the modulation of the quasi-neutral base region for our devices is minimal.

The pnp device does not exhibit impact ionization, because the ionization rate of holes is about three orders of magnitude lower than that of electrons at a fixed electrical field[7]. As a consequence, the base current of pnp devices is only affected by impact ionization at much higher $\left|V_{c b}\right|$. The small decrease of the base current, found for the pnp device, is caused by a reduced hole recombination in the quasi-neutral base region.

\section{CONCLUSIONS}

The importance of recombination in the quasi-neutral base has been measured on $n p n$ and pnp polysilicon emitter transistors with a deliberately grown interfacial oxide. In the case of pnp devices, minor deviation of the base current

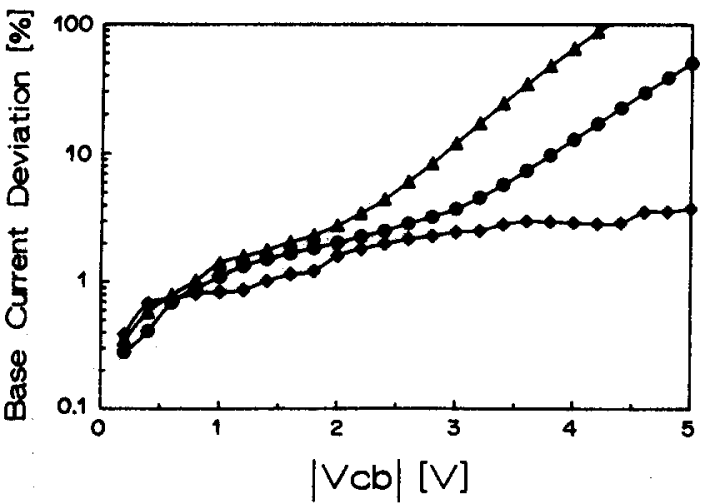

Fig. 3. The measured base current deviation as a function of the absolute collector-base voltage for the npn transistor with the high collector dose (A), the low collector dose (O) and the pnp transistor $(\bullet)$. 
could be detected. Two regions can be distinguished for the npn devices: In the first region (low $V_{c b}$ ), a small deviation of the base current is caused by reduced electron recombination in the quasi-neutral base. In the second region (higher $\left.V_{c b}\right)$, an exponential dependence is found, which is due to impact ionization in the collector-base depletion region. It can be concluded that a deliberately grown interfacial oxide considerably suppresses the base current. As a result, carrier recombination in the quasi-neutral base may become important. Simulations reveal that $20 \%$ of the base current can arise from base recombination. The influence of impact ionization in the collector-base space charge region, however, becomes important at lower reverse collector-base bias.

Faculty of Electrical Engineering

University of Twente, P.O. Box 217
RUTGer C. WiJbURG FREDERIK W. RAGAY

\section{REFLRENCES}

1. Z. Yu, B. Rioco and R. W. Dutton, IEEE Trans. Electron Devices 31, 773 (1984).

2. I. R. C. Post and P. Ashburn, Proc. ESSDERC, pp. 453-456 (1989).

3. P. Ashburn, D. J. Roulston and C. R. Selvakumar, IEEE Trans. Electron Devices 34, 1346 (1987).

4. K. Sakui, T. Hasegawa, T. Fuse, T. Seshita, S. Aritome, S. Watanabe, K. Ohuchi and F. Masuoka, Jap. J. appl. Phys. 12, L2150 (1989).

5. P. Lu and T. Chen, IEEE Trans. Electron Devices 36, 1182 (1989).

6. H. C. Poon and J. C. Meckwood, IEEE Trans. Electron Devices 19, 90 (1972).

7. S. M. Sze, Physics of Semiconductor Devices, 2nd Edn, p. 47. Wiley-Interscience, New York (1981).

8. G. R. Wolstenholme, N. Jorgensen, P. Ashburn and G. R. Booker, J. appl. Phys. 61, 225 (1987). 